\title{
Agôn
}

Revue des arts de la scène

5 | 2012

L'entrée en scène

\section{Entrée en scène, entrée en acte}

\section{Marc Douguet}

\section{(2) OpenEdition \\ Journals}

\section{Édition électronique}

URL : http://journals.openedition.org/agon/2346

DOI : 10.4000 /agon.2346

ISSN : 1961-8581

\section{Éditeur}

Association Agôn

\section{Référence électronique}

Marc Douguet, « Entrée en scène, entrée en acte », Agôn [En ligne], 5 | 2012, mis en ligne le 25 janvier 2013, consulté le 30 avril 2019. URL : http://journals.openedition.org/agon/2346 ; DOI : 10.4000/ agon. 2346

Ce document a été généré automatiquement le 30 avril 2019.

Association Agôn et les auteurs des articles 


\title{
Entrée en scène, entrée en acte
}

\author{
Marc Douguet
}

1 Le théâtre français du XVII ${ }^{\text {e }}$ siècle présente des constantes stylistiques et dramaturgiques très fortes. L'apparition de ces caractéristiques s'est parfois accompagnée de débats et d'analyses théoriques qui leur ont donné le statut de véritables règles d'écriture, destinées à conférer aux pièces la plus grande efficacité possible. Les plus connues de ces règles d'écriture sont celles qui ont suscité le plus de débats: elles concernent l'intrigue, qui doit reposer sur une action unique et unifiée, ne doit pas durer plus d'une journée et doit se dérouler dans un espace restreint aux limites d'une ville ou d'un palais.

2 Cependant, l'adaptation scénique de cette intrigue, la manière dont elle s'incarne en une succession de scènes et dont elle acquiert une forme spécifiquement théâtrale, présentent également des invariants. Ces règles concernent la disposition des scènes et la circulation des personnages, leurs mouvements d'entrée et de sortie. Elles sont passées presque inaperçues faute d'avoir été moins enfreintes et d'avoir suscité moins de débats théoriques, mais elles représentent des caractéristiques tout aussi essentielles du théâtre classique.

3 Dans une pièce classique, le nombre de personnages est relativement réduit (généralement moins d'une dizaine), mais ceux-ci ne cessent d'entrer, de sortir et de revenir sur scène. La plupart des entrées en scène sont donc des retours sur scène : il est très rare qu'un personnage entre en scène après le deuxième acte sans être déjà apparu au cours des actes précédents. C'est à travers ces aller-retour que l'action évolue.

Or, pour être nombreux, ceux-ci sont néanmoins limités par une règle tacite: un personnage ne peut pas entrer en scène deux fois au cours du même acte (c'est-à-dire qu'il ne peut pas revenir sur scène tant qu'un entracte n'a pas eu lieu). C'est à travers cette singularité du théâtre classique que l'on abordera le problème de l'entrée en scène, que l'on traitera donc d'un point de vue dramaturgique: il s'agira de s'intéresser à l'entrée en scène du personnage, et non de l'acteur, et à la manière dont celle-ci s'intègre à l'univers fictionnel de la pièce, au rôle qu'elle joue dans la construction de l'intrigue.

5 Plus que toute autre règle, celle-ci ressemble à une "règle du jeu »: il faut "attendre " l'entracte pour être " autorisé » à entrer de nouveau. Elle contribue à donner aux actes leur silhouette caractéristique. 
Le corpus classique est particulièrement unifié de ce point de vue. On prendra, au hasard, deux exemples : Rodogune de Corneille (1644-1645 ${ }^{1}$ ) et Andromaque de Racine (1667). Le tableau d'occupation scénique de ces deux pièces met clairement en évidence un schéma de circulation des personnages que l'on retrouverait dans n'importe quelle autre pièce de la période (même si les comédies présenteraient sans doute plus d'irrégularités que les tragédies).

\section{Rodogune}

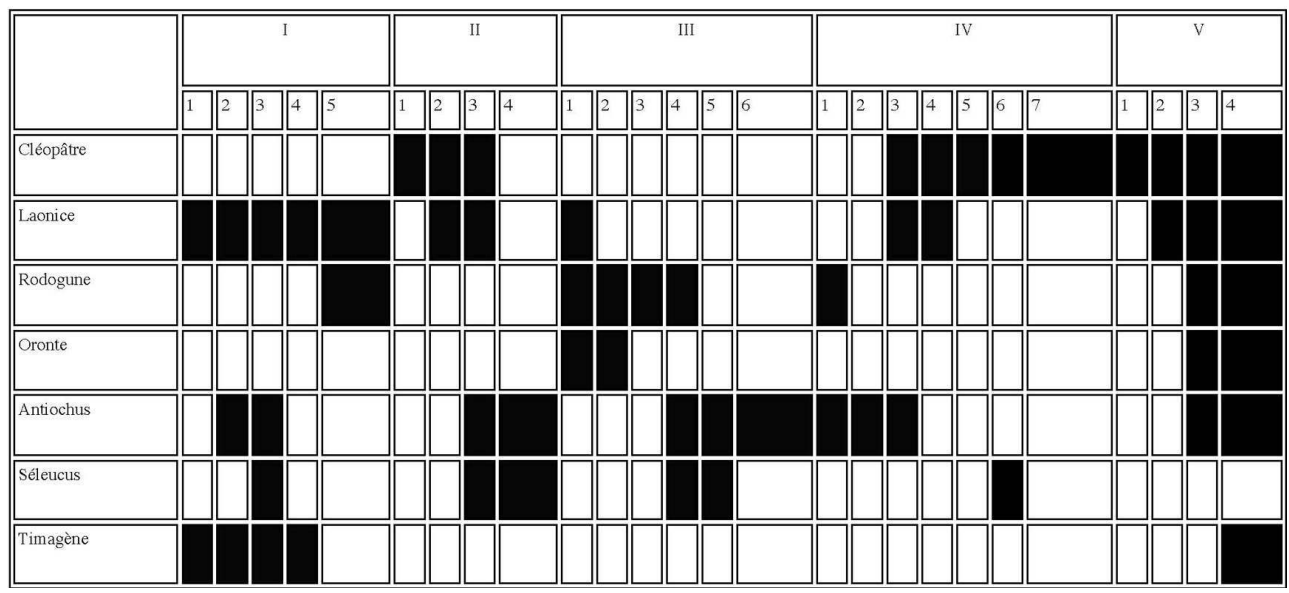

Marc Douguet

Andromaque

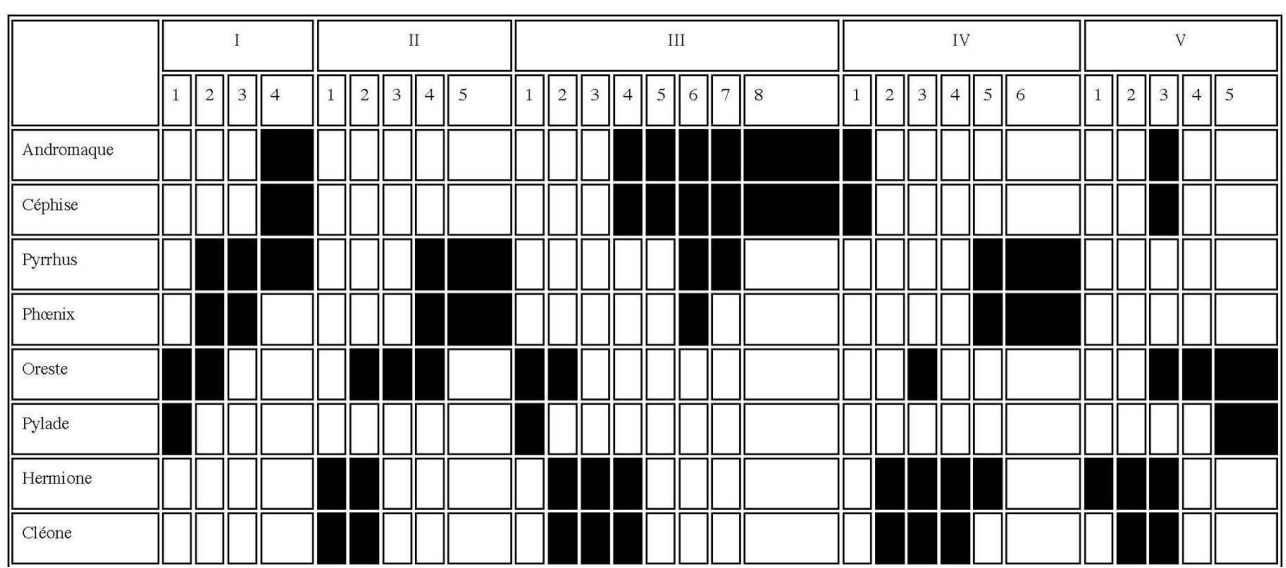

Marc Douguet

7 L'acte apparaît comme un bloc continu et compact. Pour filer la métaphore de la règle du jeu, et imaginer les personnages comme des joueurs qui seraient libres de leurs mouvements, on peut dire que, à chaque acte, chaque personnage " peut » être présent sur scène pendant un certain temps, mais que ce temps n'est pas fractionnable: le personnage «doit» dire ce qu'il a à dire et rencontrer les personnes qu'il «doit » rencontrer sans avoir la « possibilité » de repasser en coulisses. Entrer dans l'espace de la scène, c'est apparaître dans l'étendue temporelle de l'acte.

8 À partir de années 1640, la majorité des pièces présente une dispositio comparable à celle de Rodogune et d'Andromaque, comme si la «fausse sortie » que constitue le retour d'un personnage sur scène au cours d'un acte était frappée d'interdit. 
Il y a là sinon une règle, du moins une régularité dont il faut rendre compte. Pour cela, on doit s'interroger sur les enjeux propres de ces entrées en scène particulières que sont les retours sur scène de personnages précédemment sortis. Que se passe-t-il, précisément, entre leur sortie et leur retour?

10 Il y a deux manières de répondre à cette question : soit on se place du côté du personnage, et l'on cherche alors à savoir en quoi ce double mouvement participe à la construction de l'intrigue (que fait le personnage entre sa sortie et son retour, pendant le temps qu'il est absent ?) ; soit on se place du côté du spectateur, et l'on se demande alors ce qu'il advient de la représentation entre ces deux mêmes moments (sont-ils ou non séparés par un entracte?)

\section{Du côté du personnage}

11 Quand un personnage revient sur scène, deux cas de figure sont envisageables, qui impliquent chacun une manière différente de faire avancer l'intrigue : soit le personnage qui revient est censé avoir participé à une action quand il n'était pas sur scène, soit il est resté inactif. La question de l'entrée en scène est donc intimement liée à la question du hors-scène : comment décrire cet espace ? Que font les personnages lorsqu'ils ne sont pas visibles?

\section{Le champ de bataille}

Le premier cas de figure est donc celui où le personnage qui revient a participé à une action qui est supposée avoir eu lieu en coulisses. Il peut en avoir été simplement témoin : l'important est qu'un événement a eu lieu et qu'il revient l'annoncer. Dans ce cas, l'entrée a une fonction de péripétie : c'est elle qui apporte un élément nouveau, constitue un coup de théâtre. La scène qu'elle ouvre sera essentiellement occupée par les réactions des autres personnages à l'annonce de cet événement.

Dans Rodogune, Cléopâtre sort de scène à la fin de l'acte IV et y revient au début de l'acte $\mathrm{V}$ : mais elle est loin de rester inactive durant cette absence puisqu'elle tue Séleucus. Dans Andromaque, Oreste sort de scène à la scène 3 de l'acte IV, mais quand il y réapparaît à la scène 3 de l'acte $V$, il a tué Pyrrhus. Ces deux actions sont censées avoir lieu en coulisses : Cléopâtre et Oreste, bien qu'absents de scène et invisibles, ont contribué à renverser la situation.

Diderot a insisté sur l'importance de ces actions non représentées, et sur la nécessité de les traiter sur le même plan que celles qui sont jouées sur scène. Elles garantissent, pour lui, la continuité de l'intérêt dramatique: le personnage ne doit pas cesser d'exister quand il sort de scène.

Puisque l'action ne s'arrête point il faut que lorsque le mouvement cesse sur la scène, il continue derrière. Si les personnages reparaissaient et que l'action ne fût pas plus avancée que quand ils ont disparu, ils se seraient tous reposés ou ils auraient été distraits par des occupations étrangères ; deux suppositions contraires, sinon à la vérité, du moins à l'intérêt. ${ }^{2}$

15 Ce cas de figure est peut-être le plus intuitif et le plus satisfaisant pour un esprit réaliste. Il implique une conception bien précise de la représentation théâtrale. L'univers fictionnel de la pièce est en partie visible (la scène), et en partie invisible (le hors-scène), mais ces deux espaces possèdent exactement les mêmes propriétés : ils sont l'un comme 
l'autre susceptibles d'accueillir des actions, ils ont le même statut, le même degré de " réalité ». L'entrée en scène fait passer le personnage de l'un à l'autre comme on franchit une simple porte. Les actions qui se déroulent en dehors seront racontées, et non montrées, mais simplement parce qu'il est impossible de tout représenter en même temps.

\section{La salle d'attente}

Cependant, ce type de retour sur scène est loin d'être le plus répandu. Le modèle promu par Diderot est postérieur à la dramaturgie classique, et l'on aurait tort de l'appliquer de manière anachronique. La plupart du temps, dans les pièces classiques, les personnages qui reviennent sur scène, bien loin de venir annoncer l'issue d'une action accomplie en coulisses, sont incapables de rendre compte de ce qu'ils ont fait : ils sont restés inactifs, et si l'action a progressé pendant leur absence, c'est sans qu'ils y aient participé.

Reprenons Rodogune comme exemple. Antiochus et Séleucus sortent de scène à la fin de l'acte II. Ils ne réapparaissent qu'à la scène 4 de l'acte suivant. Durant leur absence, l'action progresse : Rodogune apprend que Cléopâtre veut sa mort, et décide d'éprouver l'amour que lui portent les deux frères. Mais ces événements ont lieu sur scène. Rien n'est dit de ce qui se passe hors-scène, et notamment de ce que font Antiochus et Séleucus : quand ceux-ci reviennent sur scène, ils reprennent l'action au point où ils l'avaient laissée, sans avoir conscience des péripéties dont le spectateur vient d'être le témoin.

Ce phénomène est omniprésent dans le théâtre classique. Andromaque ne fait pas exception. Oreste sort de scène à la fin de la scène 2 de l'acte I et y revient à la scène 2 de l'acte II, et l'on serait bien en peine de dire ce qu'il fait durant son absence. Là encore, le passage par le hors-scène correspond à un temps d'inactivité. Entre sa sortie et son retour, des scènes décisives se jouent (notamment la rencontre entre Andromaque et Pyrrhus), mais, du point de vue d'Oreste, la situation n'a pas changé : celui-ci est resté sur l'idée que Pyrrhus refuse de livrer Astyanax par amour pour Andromaque, et il peut donc espérer épouser Hermione.

Dans un passage du Discours sur les trois unités où il s'appuie sur un autre exemple tiré de Rodogune (l'absence de Cléopâtre entre les scènes 3 de l'acte II et 3 de l'acte IV), Corneille a lui-même défendu cette inactivité du personnage et cette « inutilisation » du hors-scène. Pour Corneille, l'inactivité des personnages n'empêche pas la continuité de l'action, la création d'un effet d'attente et la persistance d'une tension dramatique.

Quand je dis qu'il n'est pas besoin de rendre compte de ce que font les acteurs cependant qu'ils n'occupent point la scène, je n'entends pas dire qu'il ne soit quelquefois fort à propos de la rendre, mais seulement qu'on n'y est pas obligé, et qu'il n'en faut prendre le soin que quand ce qui est fait derrière le théâtre sert à l'intelligence de ce qui se doit faire devant les spectateurs. Ainsi je ne dis rien de ce qu'a fait Cléopâtre depuis le second acte jusques au quatrième, parce que durant tout ce temps-là elle a pu ne rien faire d'important pour l'action principale que je prépare, mais je fais connaître, dès le premier vers du cinquième, qu'elle a employé tout l'intervalle d'entre ces deux derniers à tuer Séleucus, parce que cette mort fait partie de l'action. ${ }^{3}$

Il est facile d'opposer cette conception à celle de Diderot : pour Corneille, le hors-scène ne possède pas les mêmes propriétés que l'espace représenté par la scène. Il est, le plus souvent, incapable d'accueillir des événements. Les personnages ne font que le traverser, il n'existe qu'en tant que salle d'attente ou que limbes. Contre une idée reçue selon 
laquelle le théâtre classique cacherait aux yeux des spectateurs la plus grande partie de l'action et remplacerait le spectacle par le récit, on ne saurait mieux dire que, la plupart du temps, c'est sur scène que se déroule l'action. Cette action est essentiellement rhétorique, ce qui la rend moins spectaculaire, mais elle a lieu sur scène, et uniquement sur scène : elle est faite d'échanges d'information, d'argumentations, de déclarations, de révélations et de prises de décision. En sortant de scène, les personnages se mettent en réserve, ils cessent pour ainsi dire d'exister.

\section{Du côté du spectateur}

21 Dans tous les exemples que l'on a donnés, la sortie du personnage et son retour sur scène sont séparés par un entracte. Celui-ci n'intervient pas nécessairement immédiatement après la sortie ou immédiatement avant le retour sur scène, et d'autres scènes peuvent venir s'intercaler, mais l'important est que le plateau ne reste pas constamment occupé entre ces deux moments. À l'acte IV de Rodogune, Rodogune rencontre Antiochus, qui rencontre Cléopâtre, qui rencontre à son tour Séleucus, de sorte qu'une continuité très forte s'établit de la première à la dernière scène. L'entracte, au contraire, est un moment de rupture où tous les personnages sont sortis de scène, et où aucun n'est encore revenu. Or seule cette discontinuité de l'occupation de la scène rend possible une interruption de la représentation.

Cette définition formelle de l'entracte comme vide scénique est datée : elle découle de la règle de la "liaison des scènes", qui s'impose aux alentours de 1640 et qui veut que le théâtre ne reste jamais vide hormis à l'occasion des entractes et que chaque scène soit liée à la suivante par un personnage qui reste sur scène pour accueillir ceux qui $\mathrm{y}$ arrivent, garantissant ainsi la continuité de la représentation. Auparavant, ces moments de rupture et de vide pouvaient avoir lieu à l'intérieur des actes, délimitant ainsi des sortes de «tableaux » (c'est notamment le cas dans Le Cid, qui date de 1637). Il va donc de soi que quand nous parlons d'actes et d'entractes, nous nous situons dans le cadre d'une pratique qui respecte la liaison des scènes.

23 La question est donc la suivante : pourquoi a-t-on besoin de ce moment de rupture entre la sortie du personnage et son retour sur scène? Que se passerait-il si ce mouvement d'aller-retour se produisait à l'intérieur d'un même acte? Il suffit d'imaginer qu'un personnage sorte de scène et que ceux avec qui il était en train de s'entretenir y demeurent jusqu'à ce qu'il y revienne. Ce schéma en apparence si simple pose de nombreux problèmes quand on réfléchit à partir des principes de la dramaturgie classique. On conservera pour la suite de notre analyse la distinction établie précédemment (le personnage qui revient sur scène a ou non participé à une action horsscène), mais on s'interrogera cette fois sur l'effet que produit sur le spectateur, dans chacun de ces deux cas, l'absence ou la présence d'un entracte entre la sortie et le retour de ce personnage.

\section{Dilatation du temps}

Le premier cas de figure est peut-être le plus facile à imaginer. Si, par exemple, un personnage sort au début d'un acte et revient à la fin du même acte après avoir livré une bataille, tandis que le plateau aura été, pendant ce temps, occupé sans interruption par d'autres personnages, le spectateur sera forcé d'imaginer que cette bataille a duré aussi 
peu de temps que les quelques scènes qui avait lieu en même temps ou que ces scènes, auxquelles il a pourtant assisté, ont duré beaucoup plus longtemps qu'elles ne le paraissaient, ce qui aboutirait à une forme particulière de dilatation du temps.

Au contraire, si ce personnage revient après un entracte, le spectateur imaginera que c'est pendant l'entracte que la bataille a eu lieu, et que ce dernier fonctionnait comme une ellipse: la discontinuité de l'occupation scénique autorise une discontinuité temporelle et permet de supposer que les actes, séparés dans le temps réel par un intermède de quelques minutes, sont séparés, dans le temps fictionnel, par un laps de temps de plusieurs heures. L'abbé d'Aubignac, qui est avec Corneille l'autre principal théoricien de la dramaturgie classique, développe longuement ce problème :

S'il y avait toujours un acteur présent au théâtre, les spectateurs ne se pourraient pas imaginer que les acteurs qui ont disparu et souvent ont besoin de beaucoup de temps pour faire ce qu'ils veulent faire en eussent employé plus que ce qui s'en serait écoulé durant le discours ou l'action de ceux qu'ils auraient vus: par exemple, un ou deux acteurs paraissant ensemble, ou successivement, consument un demi-quart d'heure de temps à ce qu'ils font ou disent sur le théâtre; et cependant un autre acteur, qui aura disparu, aura besoin de deux heures ou environ pour exécuter ce qu'il aura proposé en sortant. Or les spectateurs ne sauraient $d u$ tout s'imaginer que ces deux heures nécessaires à celui qui est absent, se soient écoulées dans un demi-quart d'heure, durant lequel ils auront vu devant leurs yeux d'autres personnes agir, et qui auront bien employé ce temps; de sorte que quand ceux qui ont disparu reviennent au théâtre et qu'ils racontent ce qu'ils ont fait, le spectateur alors trouve étrange qu'ils aient fait tout ce qu'ils disent dans ce peu de temps qu'il aura vu les autres. C'est pourquoi les poètes ont conservé ces intervalles afin que la musique, qui n'est point partie de l'action théâtrale, facilite cette agréable illusion qu'il faut faire aux spectateurs ; car l'imagination se trompe bien plus aisément lorsque les sens ne s'y opposent point; aussi quand nous sommes quelque temps sans voir aucun acteur sur la scène, et que la représentation sensible est interrompue par un divertissement qui porte notre esprit ailleurs, nous prenons volontiers ces moments pour des heures entières. ${ }^{4}$

Le temps classique repose sur un souci obsessionnel de la vraisemblance, mais c'est l'imagination et la perception du spectateur qui sont les critères de cette vraisemblance. En quelques heures de représentation, on peut faire tenir une action durant toute une journée, pourvu que l'on trouve un moyen pour que le spectateur l'accepte ou ne s'en rende pas compte : l'entracte en est un, parce qu'il s'accompagne soit d'un changement de régime (intermède musical, par exemple), soit d'une interruption pure et simple de la représentation. Quand un personnage sort, le temps fictionnel se scinde : on a d'une part le temps de l'action visible qui a lieu sous les yeux des spectateurs; d'autre part le temps de l'action invisible qui est supposée se dérouler en coulisses. Le spectateur est choqué si le deuxième doit s'aligner sur le premier. L'entracte, pendant lequel l'action visible cesse, annule cette scission, et rappelle en revanche la différence irréductible entre le temps fictionnel dans son ensemble et le temps réel où évoluent les spectateurs : il fonctionne comme une sorte de « remise à zéro » des horloges fictionnelles.

Dans Rodogune, comme on l'a vu, c'est entre sa sortie à la toute fin de l'acte IV («Allons chercher le temps d'immoler mes victimes, / Et de me rendre heureuse à force de grands crimes. ${ }^{5}$ ) et son retour sur scène au tout début de l'acte V ("Enfin, grâces aux Dieux, j'ai moins d'un ennemi, / La mort de Séleucus m'a vengée à demi. $\left.{ }^{6} \|\right)$ que Cléopâtre assassine Séleucus. Cet événement prend un certain temps, mais il a lieu pendant un entracte, et le spectateur est libre de supposer que la durée réelle de celui-ci (quelques minutes) est inférieure à sa durée fictionnelle. 
Andromaque, Pyrrhus sort de scène à la fin de l'acte IV pour se rendre au temple où il doit épouser Andromaque («Andromaque m'attend, Phœnix, garde son fils. ${ }^{7}$ ») À la scène 3 de l'acte $\mathrm{V}$, soit une centaine de vers plus tard, Oreste annonce que Pyrrhus est mort ("Madame, c'en est fait. ${ }^{8}$ ») Entre la sortie de Pyrrhus et le retour d'Oreste ont donc lieu le mariage d'Andromaque et de Pyrrhus et l'assassinat de ce dernier. On peut supposer que ces péripéties ont lieu en partie en même temps que le début de l'acte $\mathrm{V}$ (un monologue d'Hermione, qui est rejointe par sa confidente Cléone) mais elles ne sont de toute façon pas limitées dans le temps parce que la scène n'est pas occupée en permanence et que le spectateur interprète a posteriori l'entracte comme une ellipse, pendant laquelle le temps de l'histoire passe plus vite que le temps de la représentation.

Ainsi, il doit y avoir entracte entre la sortie d'un personnage et son retour sur scène pour, à la faveur d'une ellipse, "donner » à celui-ci le temps d'accomplir l'action qu'il doit accomplir. L'entracte classique constitue une sorte de soupape de sécurité permettant au trop-plein de temps de s'échapper.

Les exceptions à la règle ne font que confirmer cette hypothèse. Dans Nicomède (1651), Prusias et Flaminius quittent Arsinoé et sortent à la fin de la scène 5 de l'acte $V$ afin de mettre en œuvre un subtil stratagème (apaiser la révolte du peuple tout en enlevant en secret Nicomède à bord d'une galère romaine); Arsinoé est rejointe par Laodice; le plan échoue, Nicomède s'échappe, on croit que Prusias et Flaminius se sont enfuis, mais ils reviennent sur scène à la scène 8 ( « Non, non, nous revenons l'un et l'autre en ces lieux / Défendre votre gloire, ou mourir à vos yeux..$\left.^{9} »\right)$ Il se passe donc beaucoup plus de choses hors-scène que sur scène, ce qui donne l'impression que la scène entre Arsinoé et Laodice, comme dans un rêve, a duré beaucoup plus longtemps et que le temps s'est immobilisé pendant que les deux femmes se livraient à un ultime affrontement verbal en attendant l'issue de la révolte populaire.

De plus, le même problème se pose quand un messager vient raconter ce qu'a fait un personnage précédemment sorti de scène, mais qui ne revient pas en personne. C'est notamment le cas quand le héros tragique meurt, qu'il s'agisse d'un combat, d'un assassinat ou d'une exécution : l'annonce de sa mort doit être considérée comme sa dernière entrée en scène. Mais mourir prend du temps: si le héros sort et que l'on annonce sa mort quelques scènes plus loin à ceux qui étaient resté sur scène, l'impression de distorsion temporelle sera la même que s'il revenait vivant du combat. Ainsi Corneille reconnaît lui-même l'invraisemblance sur laquelle repose le cinquième acte d'Héraclius (1646-1647) :

Il est hors de doute que depuis que Phocas est sorti [...] jusqu'à ce qu'Amyntas vienne raconter sa mort, il faut plus de temps pour ce qui se fait derrière le théâtre que pour le récit des vers qu'Héraclius, Martian et Pulchérie emploient à plaindre leur malheur. ${ }^{10}$

La mort de Phocas et les péripéties qui l'entourent ont nécessairement lieu entre la sortie de Phocas $(V, 4)$ et l'entrée d'Amyntas $(V, 6)$ qui annonce sa mort. Dans la mesure où la scène reste en permanence occupée entre ces deux moments (par Pulchérie, Héraclius et Martian), aucune ellipse n'est possible et ces événements durent donc nécessairement aussi peu de temps que la scène 5 .

Dernier acte de Nicomède, dernier acte d'Héraclius (il faudrait également citer le dernier acte de Phèdre, où les péripéties qui entrainent la mort d'Hippolyte sont limitées en amont par la sortie d'Hippolyte et en aval par l'entrée de Théramène) : ces phénomènes de distorsion temporelle se rencontrent presque uniquement au cinquième acte. Il s'agit 
moins d'une exception que d'une convention spécifique dont les dramaturges sont conscients. Corneille réclame en effet un statut à part pour le dernier acte :

J'estime toutefois que le cinquième, par un privilège particulier, a quelque droit de presser un peu le temps, en sorte que la part de l'action qu'il représente en tienne davantage qu'il n'en faut pour sa représentation. La raison en est que le spectateur est alors dans l'impatience de voir la fin, et que quand elle dépend d'acteurs qui sont sortis du théatre, tout l'entretien qu'on donne à ceux qui y demeurent en attendant de leurs nouvelles ne fait que languir et semble demeurer sans action ${ }^{11}$. théorisée dans le cadre de la réflexion sur l' « unité de jour » et parce qu'elle est utilisée de manière systématique aux XVIII ${ }^{e}$ et XIX ${ }^{e}$ siècles, où chaque entracte correspond à une ellipse plus ou moins grande. Pourtant, cette utilisation est minoritaire au XVIII ${ }^{e}$ siècle. Comme on l'a vu, dans la dramaturgie classique, les personnages sont le plus souvent inactifs quand ils ne sont pas sur scène : l'entracte qui sépare leur sortie de leur retour sur scène ne peut donc se charger d'une fonction d'ellipse, et les actes s'enchaînent sans discontinuité temporelle.

l'espace que dans le temps, et il est, dans toutes les dramaturgies, un moment privilégié pour déplacer l'action d'un lieu à un autre, que ce changement se traduise par un changement de décor ou soit le résultat d'une simple convention. Certes, le modèle du changement de lieu peut sembler contre-intuitif dans le cas de la dramaturgie classique : les dramaturges, loin de l'expliciter, ont tout fait pour le faire oublier et l'on est habitué à lire le théâtre classique à travers le prisme de l'unité de lieu. Mais, pour les classiques, l'unité de lieu signifiait avant tout ne pas sortir des limites d'une ville, d'un palais, d'une maison. Elle n'impliquait pas nécessairement de se limiter à une seule salle ou de ne pas changer de décor. De plus, voir dans les entractes des changements de lieu est la seule manière satisfaisante de rendre compte des mouvements des personnages.

Il est facile de s'en convaincre en reprenant nos deux pièces pour exemples. Entre leur sortie à la fin de l'acte II et leur retour à la scène 4 de l'acte III, Séleucus et Antiochus, on l'a vu, sont inactifs. Mais on remarque que la raison de leur sortie est la même que celle de leur retour sur scène. Les deux frères, à qui leur mère Cléopâtre vient de demander de tuer Rodogune, dont ils sont tous deux épris, sortent pour aller trouver cette dernière afin de lui demander de choisir celui des deux qu'elle préfère ( Allons la voir, mon frère, et demeurons unis / C'est l'unique moyen de voir nos maux finis. / Je forme un beau 
dessein que son amour m'inspire. ${ }^{12}$ ») Et à la scène 4 de l'acte suivant, comme annoncé, ils entrent en scène pour parler à Rodogune, qui occupe quant à elle la scène depuis le début de l'acte ( $«$ Ne vous offensez pas, Princesse, de nous voir / De vos yeux à vous-même expliquer le pouvoir. $\left.{ }^{13} »\right)$

Où cela est-il censé se passer? On peut imaginer que l'ensemble de ces scènes a lieu dans le même vestibule du palais de Cléopâtre, un lieu où tous les personnages pourraient se trouver avec la même vraisemblance. Cependant, cette hypothèse présente deux inconvénients. D'une part, cela implique que les deux frères quittent ce lieu à la fin de l'acte II et y reviennent à la scène 4 de l'acte III : ils vont chercher Rodogune ailleurs, ne la trouvent pas et reviennent dans le lieu qu'ils ont quitté quelques scènes auparavant et qui a été entre temps occupé par la personne même qu'ils cherchaient à rencontrer. Il y a là un étrange chassé-croisé. D'autre part, s'il est facile de rendre compte de la présence des deux frères dans ce lieu à la fin de l'acte II (ils y ont été convoqués par leur mère), il est difficile de rendre compte de celle de Rodogune au début de l'acte III : pourquoi y vientelle alors que personne ne s'y trouve ? Son dialogue avec Laonice relève plus de la sphère privée et aurait plus sa place dans l'intimité d'un cabinet.

En revanche, si l'on suppose qu'un changement de lieu intervient à la faveur de l'entracte, ce chassé-croisé disparaitt et il devient plus facile de comprendre les mouvements des personnages: l'acte II se déroule dans le lieu où Cléopâtre reçoit ses fils, et l'acte III s'ouvre sur l'appartement où Rodogune s'est retirée; en passant d'un acte à l'autre, Antiochus et Séleucus passent également d'un lieu à un autre. La sortie et le retour forment en réalité un seul mouvement parfaitement rectiligne : changer de lieu pour aller trouver quelqu'un. Aucune didascalie ne confirme cette discontinuité spatiale, mais rien ne l'infirme non plus: la didascalie liminaire indique simplement «La scène est à Séleucie, dans le palais royal ». Par ailleurs, Corneille lui-même autorise cette lecture. En effet, dans le passage du Discours sur les trois unités où il expose sa conception de l'unité de lieu, il analyse la structure spatiale de Rodogune:

Je pourrais en dire ce que j'ai dit dans Cinna, où en général tout se passe dans Rome, et en particulier moitié dans le cabinet d'Auguste, et moitié chez Émilie. Suivant cet ordre, le premier acte de cette tragédie serait dans l'antichambre de Rodogune, le second dans la chambre de Cléopâtre, le troisième dans celle de Rodogune. ${ }^{14}$

Il ne s'agit pas là d'un cas isolé : le même phénomène se rencontre entre les actes I et II et entre les actes III et IV de la même pièce, et, de manière très claire, dans Andromaque. Comme on l'a vu, Oreste ne fait rien entre le moment où il remplit son rôle d'ambassadeur auprès de Pyrrhus (scène 2 de l'acte I) et celui où il réapparaît pour convaincre Hermione de l'épouser (scène 2 de l'acte II). Mais là encore, ce qui justifie son départ justifie également son retour sur scène. En effet, Pyrrhus le congédie sur ces mots :

Vous pouvez cependant voir la fille d'Hélène.

Du sang qui vous unit je sais l'étroite chaîne.

Après cela, Seigneur, je ne vous retiens plus,

Et vous pourrez aux Grecs annoncer mon refus. ${ }^{15}$

Une fois Oreste sorti, Phœnix se montre plus explicite et demande à Pyrrhus : « Ainsi vous l'envoyez aux pieds de sa maîtresse ${ }^{16}{ }^{\prime}$ Or, si Oreste revient sur scène à la scène 2 de l'acte II, c'est précisément pour voir Hermione, qui s'y trouve depuis le début de l'acte.

La didascalie liminaire précise "La scène est à Buthrot, ville d'Épire, dans une salle du palais de Pyrrhus. " Racine nous oblige donc à choisir le modèle d'une stricte unité spatiale, et interdit de voir dans les entractes des changements de lieu: tout ce qui se 
passe sur scène est censé se passer dans une même salle. Pourtant les mouvements des personnages et la structure spatiale qu'ils impliquent sont en tout point comparables à ceux de Rodogune et posent les même problèmes : pourquoi ce chassé-croisé entre Oreste et Hermione? Pourquoi Oreste revient-il sur ses pas pour rencontrer Hermione au lieu d'aller la voir dans son appartement ? L'ordre des scènes semble en contradiction avec la didascalie liminaire: tout comme dans Rodogune, il est plus satisfaisant et plus "économique " de supposer qu'un changement de lieu intervient à la faveur de l'entracte, que l'acte I se déroule dans un lieu (celui où Pyrrhus reçoit son ambassadeur), et l'acte II dans un autre, dans lequel on peut imaginer qu'Hermione se trouvait avant le début de l'acte, et où Oreste vient la rejoindre.

Les exemples sont innombrables où un personnage annonce qu'il va en rencontrer en un autre sur lequel s'ouvre dans un premier temps l'acte suivant avant qu'ait effectivement lieu la rencontre annoncée. Dans tous ces cas, chaque acte «ouvre» un nouvel espace. Évidemment, il ne s'agit pas de rejeter le modèle du « vestibule » : il contribue trop, par sa portée symbolique, au sens des pièces de Racine. Nous voudrions plutôt insister sur la coexistence de deux modèles contradictoires : en profondeur, ces pièces fonctionnent comme celle de Corneille, et même comme les pièces les plus irrégulières du début du siècle. Elles restent traversées par d'incessants changements de lieu. L'innovation de Racine a été de cacher ou de refouler cette multiplicité en faisant du "palais à volonté " non seulement le décor de ses tragédies, mais une image de l'enfermement des personnages.

Nous nous demandions ce que font les personnages quand ils ne sont pas sur scène. La réponse est simple : ils se déplacent pour aller rencontrer quelqu'un dans le lieu où il se trouve. En toute logique, ce mouvement est rectiligne et ne peut faire revenir le personnage sur ses pas. Il doit donc y avoir un entracte entre la sortie du personnage et son retour sur scène pour permettre au spectateur de supposer que le lieu que représente la scène a changé. Sinon, si la scène restait continuellement occupée, le réapparition d'un personnage donnerait l'impression soit que ce dernier a fait un aller-retour irrationnel, soit que l'espace s'est courbé pour aller à sa rencontre : comment la raison qui me pousse à sortir d'un lieu peut-elle m'y ramener sans qu'aucun changement n'ait eu lieu dans l'intervalle? La règle qui interdit le retour d'un personnage à l'intérieur d'un acte garantit donc la vraisemblance de la représentation de l'espace.

Cosroès (1648) de Rotrou nous fournira un contre-exemple révélateur. À la fin de la scène 1 de l'acte II, Cosroès envoie Sardarigue arrêter Syroès : «Arrêtez Syroès, et m'en rendez bon compte..$^{17}$ " Sardarigue sort, mais Syroès vient de lui-même trouver Cosroès à la scène 3. Il faut imaginer que pendant ce temps Sardarigue est quelque part en train de chercher Syroès. À la fin de cette scène, il revient pour accomplir l'ordre du roi. Cosroès est encore là et peut déclarer à Syroès en voyant entrer celui à qui il a donné l'ordre d'arrêter son fils deux scènes plus tôt : «Cependant recevez l'ordre qu'on vous apporte, / Prince, c'est de ma part. ${ }^{18}$ » Dans le même mouvement, Sardarigue sort et revient. Personne ne s'en étonne et aucune précision n'est donnée: faut-il comprendre que Sardarigue revient parce qu'il n'a pas trouvé Syroès ? Parce qu'il l'a vu venir parler à Cosroès et l'a suivi ? Parce qu'il a oublié l'ordre qu'on lui avait donné ? Cet aller-retour étrange n'est pas sans beauté et peut être interprété comme un signe du désordre et de l'atmosphère générale d'abdication qui règne dans le palais. Il possède également des raisons dramaturgiques : il faut que Sardarigue sorte pour laisser le temps à Syroès de venir de lui-même se plaindre à son père, mais il faut aussi qu'il revienne pour l'arrêter. Il n'en reste pas moins qu'il y a 
aussi là un dysfonctionnement, une anomalie dans la cohérence entre l'espace et les mouvements des personnages.

Le retour d'un personnage au cours d'un même acte est donc à l'origine de distorsions dans la représentation du temps et de l'espace. Le système classique s'en accommode parfois (notamment à l'approche du dénouement), mais il propose aussi une solution originale : chaque personnage ne pourra entrer en scène qu'une seule fois par acte. S'il vient annoncer l'issue d'une action qu'il a accomplie hors-scène, l'entracte qui précède son entrée fonctionnera comme une ellipse et permettra de résorber la trop longue durée de cette action. Si cette entrée se situe dans le prolongement de sa sortie et forme avec elle un mouvement de déplacement d'un lieu à un autre, l'entracte fonctionnera comme un changement de lieu. L'entrée en scène d'un personnage est à chaque fois la première fois qu'il apparaît dans l'acte : faire ainsi coïncider entrée en scène et "entrée en acte » permet d'exploiter les fonctions dramaturgiques de l'entracte qui précède.

\section{BIBLIOGRAPHIE}

Aubignac, abbé d', La Pratique du théâtre [1657], [éd. H. Baby], Paris, Champion, 2001

Corneille, Pierre, Trois Discours sur le poème dramatique [1660], [éd. M. Escola et B. Louvat], Paris, Flammarion, coll. «GF », 1999

Diderot, Denis, De la poésie dramatique [1758], dans Euvres complètes, [éd. J. Chouillet et A.-M. Chouillet], t. X, Paris, Hermann, 1980

\section{NOTES}

1. J'indique pour chaque pièce l'année, ou, comme ici, la saison théâtrale de la première représentation.

2. Denis Diderot, De la poésie dramatique [1758], dans CEuvres complètes, [éd. Jacques Chouillet et Anne-Marie Chouillet], t. X, Paris, Hermann, 1980, p. 385.

3. Pierre Corneille, Trois Discours sur le poème dramatique [1660], [éd. M. Escola et B. Louvat], Paris, Flammarion, coll. « GF », 1999, p. 134-135.

4. Abbé d'Aubignac, La Pratique du théâtre [1657], [éd. H. Baby], Paris, Champion, 2001, p. 351-352.

5. v. 1496-1497.

6. v. 1498-1499.

7. v. 1400 (dans l'édition de 1668).

8. v. 1501.

9. v. 1765-1766.

10. Pierre Corneille, Trois Discours sur le poème dramatique, op. cit., p. 146.

11. Id.

12. v. 749-751.

13. v. 897-898.

14. Pierre Corneille, Trois Discours sur le poème dramatique, op. cit., p. 149. 
15. V. 245-248.

16. v. 249.

17. V. 530 .

18. V. $683-684$.

\section{RÉSUMÉS}

Dans la dramaturgie classique, la circulation des personnages obéit à une règle à la fois stricte et tacite : un personnage ne peut entrer en scène deux fois au cours du même acte. Pourquoi cette adéquation entre entrée en scène et "entrée en acte "? On verra que cette règle permet de résoudre deux problèmes qui, selon les principes de la dramaturgie classique, se posent dans la représentation de l'espace et du temps : en effet, si un personnage sort de scène et y revient au cours du même acte, le spectateur aura l'impression d'une distorsion du temps (si ce personnage a participé à une action hors-scène) ou de l'espace (s'il est resté inactif durant son absence). Au contraire, le fait qu'un personnage ne puisse pas revenir sur scène tant qu'un entracte n'a pas eu lieu permet d'exploiter les fonctions d'ellipse ou de changement de lieu qui sont inhérentes à l'entracte et qui permettront de résoudre ces diverses incohérences.

\section{INDEX}

Mots-clés : dramaturgie, personnage, Corneille (Pierre), Racine (Jean)

\section{AUTEUR}

\section{MARC DOUGUET}

Doctorant allocataire

Université Paris 8 - Vincennes à Saint-Denis

marc.douguet[at]gmail.com 